PHYSICAL REVIEW D 95, 084002 (2017)

\title{
Generalized Vaidya solutions and Misner-Sharp mass for $n$-dimensional massive gravity
}

\author{
Ya-Peng $\mathrm{Hu},{ }^{1,2,3,{ }^{*}}$ Xin-Meng $\mathrm{Wu},{ }^{1, \dagger}$ and Hongsheng Zhang ${ }^{4,3,5, *}$ \\ ${ }^{1}$ College of Science, Nanjing University of Aeronautics and Astronautics, Nanjing 210016, China \\ ${ }^{2}$ Instituut-Lorentz for Theoretical Physics, Leiden University, \\ Niels Bohrweg 2, Leiden 2333 CA, The Netherlands \\ ${ }^{3}$ Key Laboratory of Theoretical Physics, Institute of Theoretical Physics, \\ Chinese Academy of Sciences, Beijing 100190, China \\ ${ }^{4}$ School of Physics and Technology, University of Jinan, \\ 336 West Road of Nan Xinzhuang, Jinan, Shandong 250022, China \\ ${ }^{5}$ Center for Astrophysics, Shanghai Normal University, 100 Guilin Road, Shanghai 200234, China
} (Received 1 December 2016; published 3 April 2017)

\begin{abstract}
Dynamical solutions are always of interest to people in gravity theories. We derive a series of generalized Vaidya solutions in the $n$-dimensional de Rham-Gabadadze-Tolley massive gravity with a singular reference metric. Similar to the case of the Einstein gravity, the generalized Vaidya solution can describe shining/absorbing stars. Moreover, we also find a more general Vaidya-like solution by introducing a more generic matter field than the pure radiation in the original Vaidya spacetime. As a result, the above generalized Vaidya solution is naturally included in this Vaidya-like solution as a special case. We investigate the thermodynamics for this Vaidya-like spacetime by using the unified first law and present the generalized Misner-Sharp mass. Our results show that the generalized Minser-Sharp mass does exist in this spacetime. In addition, the usual Clausius relation $\delta Q=T d S$ holds on the apparent horizon, which implicates that the massive gravity is in a thermodynamic equilibrium state. We find that the work density vanishes for the generalized Vaidya solution, while it appears in the more general Vaidya-like solution. Furthermore, the covariant generalized Minser-Sharp mass in the $n$-dimensional de Rham-GabadadzeTolley massive gravity is also derived by taking a general metric ansatz into account.
\end{abstract}

DOI: 10.1103/PhysRevD.95.084002

\section{INTRODUCTION}

Massive gravities are significant and fundamental extensions of the Einstein gravity, but, in opposition to our intuition, to endow a mass to the graviton is not an easy problem. In 1939, Fierz and Pauli first introduced the linear massive gravity theory [1]. Note that a massless graviton has only two polarizations, and a sound massive gravity theory generally has 5 degrees of freedom. However, the surplus 3 degrees of freedom have been proven to be intractable when the mass of graviton vanishes in the linear massive gravity [2]. To overcome this problem, one tries to introduce the nonlinear massive gravities, but a more serious problem, the Boulware-Deser ghost problem, appears [3]. Recently, the so-called de Rham-GabadadzeTolley (dRGT) massive gravity, which is a nonlinear massive gravity theory and has been shown to be ghost free, was proposed $[4-6,7,8]$. Note that in the dRGT model the reference metric is full rank, but a singular reference metric is also important [9], and the ghost problem in it is investigated in Refs. [10,11]. Moreover, according to the AdS/CFT correspondence [12-14], many clues have

\footnotetext{
*huyp@nuaa.edu.cn

†wuxm@nuaa.edu.cn

†hongsheng@shnu.edu.cn
}

shown that the massive graviton in the bulk is related to some interesting effects of the dual field which resides on the UV boundary of an asymptotical anti-de Sitter (AdS) spacetime, i.e., the effects like a lattice to deduce the momentum dissipation [9,15-17]. Much research about the dRGT massive gravity has been done [9-11,15-31].

Among this research, one interesting issue is to find out exact solutions in the dRGT massive gravity [18-24]. Usually, we assume some symmetries of the spacetime when we seek a new solution. The translation invariance along a timelike Killing vector is one of the most important symmetries, but in some violent astrophysical processes, or when the mass of the matters surrounding the central celestial bodies are not negligible, such an assumption may no longer be reliable. However, finding an exact dynamical solution describing these realistic processes has proven to be an intricate issue.

Vaidya found an important dynamical toy model for a spherically symmetric spacetime [32],

$$
d s^{2}=-\left(1-\frac{2 M(v)}{r}\right) d v^{2}+2 d v d r+r^{2} d \Omega_{2}^{2},
$$

where $M(v)$ is the mass parameter, $d \Omega_{2}^{2}$ is the metric of the 2-sphere, and the stress tensor of the matter field is given by $T_{a b}=\mu l_{a} l_{b}$. Here, $l_{a}=(d v)_{a}$ in the above coordinates 
$\left(v, r, x^{i}\right)$, and $\mu$ is the energy density. This solution is well known as the Vaidya solution. Note that the Vaidya solution describes a spherically symmetric spacetime sourced by massless particles (not quanta of the Maxwell field which are called the pure radiation). In addition, since $M(v)$ is an undetermined function in the Vaidya metric, in principle, it can describe an arbitrary spherically symmetric energy flow from the central star. When $M(v)=$ constant, it comes back to the Schwarzschild spacetime, and when $M(v)=0$, it degenerates to the Minkowski spacetime. It should be emphasized that the Vaidya solution is an important solution since it encodes some essential properties of the dynamical spherically symmetric spacetimes, while remaining simple enough to handle. Therefore, in our paper, the first task is to generalize the above Vaidya solution to a more general case, i.e., the exact generalized Vaidya and Vaidya-like solutions in the $n$-dimensional spacetime with maximally symmetric $(n-2)$-subspace in the dRGT massive gravity. The metric ansatz reads

$$
d s^{2}=-f(v, r) d v^{2}+2 d v d r+r^{2} \gamma_{i j} d x^{i} d x^{j},
$$

where $\gamma_{i j}$ is the metric on a $(n-2)$-dimensional constant curvature space $\mathcal{N}$ with its sectional curvature $k= \pm 1,0$, and the two-dimensional $\mathcal{T}$ spanned by $(v, r)$ has the metric $h_{a b}$. In addition, during obtaining the generalized dynamical solutions, we first adopt the pure radiation as the matter field. Then, we extend the matter field to a more general case $[33,34]$ and then obtain a generalized Vaidya-like solution, in which the generalized Vaidya solution is included as a special case. For the generalized Vaidya solution in dRGT massive gravity, we find that it is consistent with the result in some previous works in which the corresponding static solution has been found [18].

As important progress, black hole thermodynamics (more generally gravithermodynamics) significantly boosts our understanding of gravity theory. It is even treated as a critical probe of the quantum gravity theory. Gravithermodynamics is well established in stationary spacetime. For dynamical spacetimes, there is still no generally accepted theory yet. The first difficulty is that some key physical concepts, including temperature, entropy, horizon, etc., become subtle. The second difficulty is that it is hard to define a reversible process in a dynamical spacetime. However, some research has shown that the unified first law is a nice approach in gravithermodynamics if the spacetime has a maximally symmetric subspace, since usually it can be directly derived from the field equation itself $[10,34-38]$ in such spacetimes. Thus, one can apply it in a dynamical spacetime without essential obstructions for these spacetimes. In our paper, we apply the unified first law to investigate the thermodynamics of the above generalized dynamical solutions in dRGT massive gravity. Note that the Misner-Sharp mass is a significant quantity in the unified first law. In Einstein's general relativity, the Misner-Sharp mass always exists [10,34-38]. In addition, since it encodes rich information of the corresponding gravity field [39], one can obtain a series of exact solutions through thermodynamic approaches from the Misner-Sharp mass [40]. However, the generalized Misner-Sharp mass may be absent in some modified gravity like $f(R)$ gravity [41,42].

In our case, by using the unified first law, we find that the generalized Misner-Sharp mass does exist for the above generalized dynamical solutions and obtain the first law of thermodynamics on the apparent horizon for these generalized dynamical solutions. In addition, the usual Clausius relation $\delta Q=T d S$ holds on the apparent horizon, which implies that the dRGT massive gravity is in a thermodynamic equilibrium state $[10,41,43,44]$. It should be emphasized that the existence of the Misner-Sharp mass in some special solutions does not always imply the existence of it in the corresponding gravity theory. For example, the Misner-Sharp mass exists in the Friedmann-RobertsonWalker (FRW) solution and static solution in $f(R)$ gravity. However, it does not always exist in a general spherically symmetric spacetime in $f(R)$ gravity [41,42]. Essentially, the generalized Misner-Sharp mass is a conserved charge of the spacetime corresponding to the Kodama vector (reduced to a Killing one in stationary spacetime), which depends on the gravity theory in consideration $[36,41,42]$. The integrability of such a conserved charge, and thus the existence of the generalized Misner-Sharp mass, is a nontrivial problem. Therefore, we need further study the existence of the generalized Misner-Sharp mass in a general spacetime with maximally symmetric subspaces. We show that the generalized Misner-Sharp mass in the $n$-dimensional dRGT massive gravity indeed exists, and the covariant form has also been obtained; i.e., the result is not constrained to any special solution.

This paper is organized as follows. In Sec. II, we first obtain the generalized Vaidya solution in the dRGT massive gravity and then consider a more general matter field to obtain a generalized Vaidya-like solution. In Sec. III, we use the unified first law to investigate the thermodynamics of these generalized dynamical solutions. Our results show that the generalized Misner-Sharp mass exists in these solutions. In Sec. IV, we further derive the covariant generalized Misner-Sharp mass for the $n$-dimensional dRGT massive gravity by considering the more general metric ansatz and matter fields. Finally, we draw the conclusions and discussions in Sec. V.

\section{GENERALIZED DYNAMICAL SOLUTIONS IN THE $N$-DIMENSIONAL MASSIVE GRAVITY}

In this section, we explore the generalized dynamical solutions in the $n$-dimensional dRGT massive gravity. The action of the dRGT massive gravity in an $n$-dimensional spacetime with a cosmological constant $\Lambda=-\frac{(n-1)(n-2)}{2 \ell^{2}}$ reads $[9,18]$ 


$$
\begin{aligned}
S= & \frac{1}{16 \pi G} \int d^{n} x \sqrt{-g}\left[R+\frac{(n-1)(n-2)}{\ell^{2}}\right. \\
& \left.+m^{2} \sum_{i}^{4} c_{i} \mathcal{U}_{i}(g, f)\right]
\end{aligned}
$$

where $f$ is a constant symmetric tensor, which is usually called the reference metric; $c_{i}$ and $\ell$ are constants; and $\mathcal{U}_{i}$ are symmetric polynomials of the eigenvalues of the $n \times n$ matrix $\mathcal{K}_{\nu}^{\mu} \equiv \sqrt{g^{\mu \alpha} f_{\alpha \nu}}$

$\mathcal{U}_{1}=[\mathcal{K}]$,

$\mathcal{U}_{2}=[\mathcal{K}]^{2}-\left[\mathcal{K}^{2}\right]$,

$\mathcal{U}_{3}=[\mathcal{K}]^{3}-3[\mathcal{K}]\left[\mathcal{K}^{2}\right]+2\left[\mathcal{K}^{3}\right]$,

$\mathcal{U}_{4}=[\mathcal{K}]^{4}-6\left[\mathcal{K}^{2}\right][\mathcal{K}]^{2}+8\left[\mathcal{K}^{3}\right][\mathcal{K}]+3\left[\mathcal{K}^{2}\right]^{2}-6\left[\mathcal{K}^{4}\right]$.

The square root in $\mathcal{K}$ means $(\sqrt{A})^{\mu}{ }_{\nu}(\sqrt{A})^{\nu}{ }_{\lambda}=A^{\mu}{ }_{\lambda}$ and $[\mathcal{K}]=K_{\mu}^{\mu}=\sqrt{g^{\mu \alpha} f_{\alpha \mu}}$.

From the action and considering the matter fields, the equations of motion are

$$
\begin{aligned}
\mathcal{G}_{\mu \nu} & \equiv R_{\mu \nu}-\frac{1}{2} R g_{\mu \nu}-\frac{(n-1)(n-2)}{2 \ell^{2}} g_{\mu \nu}+m^{2} \chi_{\mu \nu} \\
& =8 \pi G T_{\mu \nu},
\end{aligned}
$$

where

$$
\begin{aligned}
\chi_{\mu \nu}= & -\frac{c_{1}}{2}\left(\mathcal{U}_{1} g_{\mu \nu}-\mathcal{K}_{\mu \nu}\right)-\frac{c_{2}}{2}\left(\mathcal{U}_{2} g_{\mu \nu}-2 \mathcal{U}_{1} \mathcal{K}_{\mu \nu}+2 \mathcal{K}_{\mu \nu}^{2}\right) \\
& -\frac{c_{3}}{2}\left(\mathcal{U}_{3} g_{\mu \nu}-3 \mathcal{U}_{2} \mathcal{K}_{\mu \nu}+6 \mathcal{U}_{1} \mathcal{K}_{\mu \nu}^{2}-6 \mathcal{K}_{\mu \nu}^{3}\right) \\
& -\frac{c_{4}}{2}\left(\mathcal{U}_{4} g_{\mu \nu}-4 \mathcal{U}_{3} \mathcal{K}_{\mu \nu}+12 \mathcal{U}_{2} \mathcal{K}_{\mu \nu}^{2}\right. \\
& \left.-24 \mathcal{U}_{1} \mathcal{K}_{\mu \nu}^{3}+24 \mathcal{K}_{\mu \nu}^{4}\right) .
\end{aligned}
$$

In this article, we will investigate the generalized dynamical solutions in the $n$-dimensional spacetime with a maximally symmetric inner space in the dRGT massive gravity, and the metric ansatz is just (2). For this metric ansatz, we take the reference metric as in Ref. [18],

$$
f_{\mu \nu}=\operatorname{diag}\left(0,0, c_{0}^{2} \gamma_{i j}\right),
$$

with $c_{0}$ is a positive constant. Thus,

$$
\begin{aligned}
{[\mathcal{K}] } & =\frac{n-2}{r} c_{0}, & {\left[\mathcal{K}^{2}\right] } & =\frac{n-2}{r^{2}} c_{0}^{2}, \\
{\left[\mathcal{K}^{3}\right] } & =\frac{n-2}{r^{3}} c_{0}^{3}, & {\left[\mathcal{K}^{4}\right] } & =\frac{n-2}{r^{4}} c_{0}^{4},
\end{aligned}
$$

where the symmetric polynomials become

$$
\mathcal{U}_{1}=\frac{(n-2) c_{0}}{r}
$$

$$
\mathcal{U}_{2}=\frac{(n-2)(n-3) c_{0}^{2}}{r^{2}}
$$

$$
\begin{gathered}
\mathcal{G}_{v}^{v}=\mathcal{G}_{r}^{r}=\Lambda+\frac{n-2}{2}\left[\frac{\left(r^{n-3} f\right)^{\prime}-(n-3) r^{n-4} k-c_{1} c_{0} m^{2} r^{n-3}-(n-3) c_{2} c_{0}^{2} m^{2} r^{n-4}-(n-3)(n-4) c_{3} c_{0}^{3} m^{2} r^{n-5}}{r^{n-2}}\right. \\
-\frac{\left.(n-3)(n-4)(n-5) c_{4} c_{0}^{4} m^{2} r^{n-6}\right]}{\left.r^{n-2}\right]} \\
\mathcal{G}_{j}^{i}=\delta_{j}^{i}\left[\Lambda+\frac{\left(r^{n-3} f\right)^{\prime \prime}-(n-3)(n-4) r^{n-5} k-(n-3) c_{1} c_{0} m^{2} r^{n-4}-(n-3)(n-4) c_{2} c_{0}^{2} m^{2} r^{n-5}}{2 r^{n-3}}\right. \\
\left.-\frac{(n-3)(n-4)(n-5) c_{3} c_{0}^{3} m^{2} r^{n-6}-(n-3)(n-4)(n-5)(n-6) c_{4} c_{0}^{4} m^{2} r^{n-7}}{2 r^{n-3}}\right], \\
\mathcal{G}_{v}^{r}=\frac{-(n-2) \dot{f}}{2 r},
\end{gathered}
$$




$$
\mathcal{G}_{r}^{v}=0,
$$

where a prime/overdot denotes the derivative with respect to $r / v$. In the following, we investigate two cases by considering different matter fields. In the first case, the generalized Vaidya solution is derived by the pure radiation in analogy to the Vaidya solution in the Einstein gravity. In the second case, we consider a more generic source matter than the usual pure radiation to obtain a more general dynamical solution, i.e., a generalized Vaidya-like solution.

\section{A. Special case: Generalized Vaidya solution}

For the pure radiation, the stress-energy tensor is given by $T_{a b}=\mu l_{a} l_{b}$, where $l_{a}=(d v)_{a}$ is expressed in the coordinates $\left(v, r, x^{i}\right)$ in (2). The components of the field equation (5) corresponding to the metric (2) present

$$
\begin{gathered}
\mathcal{G}_{v}^{v}=\mathcal{G}_{r}^{r}=\Lambda+\frac{n-2}{2} \times\left[\frac{\left(r^{n-3} f\right)^{\prime}-(n-3) r^{n-4} k-c_{1} c_{0} m^{2} r^{n-3}-(n-3) c_{2} c_{0}^{2} m^{2} r^{n-4}-(n-3)(n-4) c_{3} c_{0}^{3} m^{2} r^{n-5}}{r^{n-2}}\right. \\
\left.-\frac{(n-3)(n-4)(n-5) c_{4} c_{0}^{4} m^{2} r^{n-6}}{r^{n-2}}\right]=0, \\
\mathcal{G}_{j}^{i}=\delta_{j}^{i} \times\left[\Lambda+\frac{\left(r^{n-3} f\right)^{\prime \prime}-(n-3)(n-4) r^{n-5} k-(n-3) c_{1} c_{0} m^{2} r^{n-4}-(n-3)(n-4) c_{2} c_{0}^{2} m^{2} r^{n-5}}{2 r^{n-3}}\right. \\
\left.-\frac{(n-3)(n-4)(n-5) c_{3} c_{0}^{3} m^{2} r^{n-6}-(n-3)(n-4)(n-5)(n-6) c_{4} c_{0}^{4} m^{2} r^{n-7}}{2 r^{n-3}}\right]=0,
\end{gathered}
$$

$$
\begin{gathered}
\mathcal{G}_{v}^{r}=\frac{-(n-2) \dot{f}}{2 r}=8 \pi G \mu, \\
\mathcal{G}_{r}^{v}=0 .
\end{gathered}
$$

Note that the components $\mathcal{G}_{j}^{i}$ are not independent, because they are a linear combination of the terms of $\mathcal{G}_{v}^{v}$ and $\partial_{r} \mathcal{G}_{v}^{v}$,

$$
\begin{aligned}
\mathcal{G}_{j}^{i} & =\delta_{j}^{i}\left[\mathcal{G}_{v}^{v}+r \partial_{r} \mathcal{G}_{v}^{v} /(n-2)\right] \\
& =\delta_{j}^{i}\left[\frac{1}{(n-2) r^{n-3}} \partial_{r}\left(r^{n-2} \mathcal{G}_{v}^{v}\right)\right] .
\end{aligned}
$$

Therefore, $\mathcal{G}_{j}^{i}=0$ do not yield independent equations. From the above equation in (17), we can easily obtain the generalized Vaidya solution in the $n$-dimensional dRGT massive gravity,

$$
\begin{aligned}
f(v, r)= & k+\frac{r^{2}}{\ell^{2}}-\frac{M(v)}{r^{n-3}}+\frac{c_{0} c_{1} m^{2}}{n-2} r+c_{0}^{2} c_{2} m^{2} \\
& +\frac{(n-3) c_{0}^{3} c_{3} m^{2}}{r}+\frac{(n-3)(n-4) c_{0}^{4} c_{4} m^{2}}{r^{2}},
\end{aligned}
$$

with

$$
\mu=-\frac{(n-2) \dot{f}}{16 \pi G r}=\frac{(n-2) \dot{M}(v)}{16 \pi G r^{n-2}},
$$

which can be obtained by inserting (22) into (19), and $M(v)$ is the mass parameter. Our solution is consistent with the result in some previous works like Ref. [18]. Since if $M(v)$ is independent of $v$, i.e., a constant, and hence $f(v, r)$ can be written as $f(r)$, then after the transformation in the metric ansatz (2)

$$
d v=d t+\frac{1}{f(r)} d r
$$

the above solution (22) comes back to the static solution in $n$-dimensional spacetime found in Ref. [18].

\section{B. General case: Generalized Vaidya-like solution}

Now, we further generalize the above generalized Vaidya solution in the dRGT massive gravity to a more general case. For a general discussion of the stress-energy form constrained by the energy conditions in the Vaidya-type solutions, see Ref. [45]. Note that for the metric (2) and the reference metric (7) we have $\mathcal{G}_{r}^{r}=\mathcal{G}_{v}^{v}$, so the energymomentum tensor of the matter field should satisfy $T_{r}^{r}=T_{v}^{v}$. Certainly, the pure radiation matter discussed above satisfies the constraint. In fact, it is $T_{r}^{r}=T_{v}^{v}=0$. Therefore, if we relax this condition to $T_{i}^{i}=\sigma T_{r}^{r}=\sigma T_{v}^{v}$ (where $\sigma$ is a constant, and the equation does not sum over $i)$, then from the equation $\nabla_{\mu} T_{\nu}^{\mu}=0$ or the explicit expressions of $\mathcal{G}_{\nu}^{\mu}$ in Eqs. (13) to (15), we can derive

$$
\partial_{v} T_{v}^{v}+\partial_{r} T_{v}^{r}+\frac{n-2}{r} T_{v}^{r}=0
$$


and

$$
\partial_{r} T_{v}^{v}+\frac{(n-2)(1-\sigma)}{r} T_{v}^{v}=0 .
$$

So, for the pure radiation matter with $T_{r}^{r}=T_{v}^{v}=0$, one finds that $T_{v}^{r}$ has to be proportional to $1 / r^{n-2}$, which is consistent with the above generalized Vaidya case in Eq. (23).

Therefore, for the more general case $T_{r}^{r}=T_{v}^{v} \neq 0$ for the matter field, and hence from Eq. (26), $T_{r}^{r}$ and $T_{v}^{v}$ should satisfy

$$
T_{r}^{r}=T_{v}^{v}=\mathcal{C}(v) r^{-(n-2)(1-\sigma)},
$$

where $\mathcal{C}(v)$ is a function of $v$. In addition, the off-diagonal part of the energy-momentum tensor $T_{\nu}^{\mu}$, i.e., the component $T_{v}^{r}$, has to satisfy Eq. (25). Now, Eq. (17) is modified as

$$
\mathcal{G}_{v}^{v}=8 \pi G \mathcal{C}(v) r^{-(n-2)(1-\sigma)} .
$$

Integrating this equation, we obtain the expression of $f(v, r)$,

$$
\begin{aligned}
f(v, r)= & k+\frac{r^{2}}{\ell^{2}}+\frac{c_{0} c_{1} m^{2}}{n-2} r+c_{0}^{2} c_{2} m^{2}+\frac{(n-3) c_{0}^{3} c_{3} m^{2}}{r} \\
& +\frac{(n-3)(n-4) c_{0}^{4} c_{4} m^{2}}{r^{2}} \\
& -\frac{M(v)}{r^{n-3}}+\frac{16 \pi G}{(n-2) r^{n-3}} \mathcal{C}(v) \Theta(r)
\end{aligned}
$$

where $M(v)$ is an arbitrary function of $v$, and $\Theta(r)=\int d r r^{(n-2) \sigma}$. In detail, when $\sigma=-1 /(n-2)$,

$$
\Theta(r)=\ln (r),
$$

and in other cases,

$$
\Theta(r)=\frac{r^{(n-2) \sigma+1}}{(n-2) \sigma+1} .
$$

Note that the parameter $\sigma$ and functions $m(v)$ and $\mathcal{C}(v)$ should satisfy some consistency relations if one imposes some energy condition for the energy-momentum tensor. In addition, from Eq. (15), we have

$$
T_{v}^{r}=\tilde{\mu}=\frac{(n-2) \dot{M}(v)}{16 \pi G r^{n-2}}-\frac{\dot{\mathcal{C}}(v) \Theta(r)}{r^{n-2}},
$$

which is also consistent with Eq. (25). Therefore, we have also obtained the stress tensor of the matter field in this more general case. More precisely, we can further write the stress tensor of the matter field in this more general case as

$$
T_{a b}=\tilde{\mu} l_{a} l_{b}-P\left(l_{a} n_{b}+n_{a} l_{b}\right)+\sigma P q_{a b}
$$

where $n_{a}$ is a null vector which satisfies $l_{a} n^{a}=-1$. In coordinates $\left(v, r, x^{i}\right), l_{a}=(d v)_{a}$ and $n_{a}=f / 2(d v)_{a}-$ $(d r)_{a}$, while the tensor $q_{a b}$ is a projection operator given by $q_{a b}=g_{a b}+l_{a} n_{b}+l_{b} n_{a}$, and the quantity $P$ is the radial pressure with the form $P=\mathcal{C}(v) r^{-(n-2)(1-\sigma)}$. In addition, the metric (2) can be put into the form $g_{a b}=h_{a b}+q_{a b}$, where

$$
h_{a b}=-l_{a} n_{b}-l_{b} n_{a}
$$

is the metric of two-dimensional spacetime $\mathcal{T}$ spanned by the coordinates $(v, r)$. Certainly, in the coordinates $\left(v, r, x^{i}\right)$, the line element of $h_{a b}$ can be expressed as $-f(v, r) d v^{2}+2 d v d r$. Therefore, Eq. (29) together with Eq. (33) is a more general case with the new dynamical solution, which we call the generalized Vaidya-like solution. Obviously, the above generalized Vaidya solution is a special case of this generalized Vaidya-like solution with $\mathcal{C}(v)=0$.

\section{THERMODYNAMICS OF THE GENERALIZED DYNAMICAL SOLUTIONS}

In this section, we will investigate thermodynamics of the above generalized dynamical solutions in the dRGT massive gravity by using the unified first law, and we concentrate on the generalized Vaidya-like solution obtained in the more general case, since it naturally includes the generalized Vaidya solution as a special case. According to the unified first law, similar to the case of the Einstein gravity [35], one can formally cast Eq. (5) of the gravitational field into the form

$$
d M_{\mathrm{eff}}=A \Psi_{a} d x^{a}+W d V,
$$

where $A=V_{k} r^{n-2}$ and $V=V_{k} r^{n-1} /(n-1)$ are the area and volume of the $(n-2)$-dimensional constant curvature space $\mathcal{N}$ with radius $r, W$ is called work density defined as $W=-h^{a b} T_{a b} / 2$, and $\Psi_{a}$ is the energy supply vector with the definition $\Psi_{a}=T_{a}^{b} \partial_{b} r+W \partial_{a} r$. Here, $T_{a b}$ is the projection of the stress tensor $T_{\mu \nu}$ of matter into $h_{a b}$.

After substituting the explicit forms of generalized dynamical solutions in the dRGT massive gravity (29) and (33), we can explicitly obtain the following quantities:

$$
\begin{aligned}
& W=-P, \quad \Psi_{a}=\tilde{\mu} l_{a}, \\
& A \Psi_{a} d x^{a}+W d V=V_{k} r^{n-2} \tilde{\mu} d v-P V_{k} r^{n-2} d r \\
& \equiv X(v, r) d v+Y(v, r) d r .
\end{aligned}
$$

It is easy to check, 


$$
\frac{\partial X(v, r)}{\partial r}=\frac{\partial Y(v, r)}{\partial v},
$$

which ensures that $d M_{\text {eff }}$ is a closed form and thus qualified as the generalized Misner-Sharp mass for the above generalized dynamical solutions in the dRGT massive gravity. Moreover, the generalized Misner-Sharp mass can be easily obtained in this case,

$$
M_{\mathrm{eff}}=V_{k}\left[\frac{(n-2) M(v)}{16 \pi G}-\mathcal{C}(v) \Theta(r)\right] .
$$

Next, we will use the unified first law and generalized Misner-Sharp mass (39) to investigate the thermodynamics of the above generalized dynamical solutions on the apparent horizon $r_{A}$, where $r_{A}$ is defined as the trapped surface $h^{a b} \partial_{a} r \partial_{b} r=0$. In our case, we can easily obtain that the location of the apparent horizon $r_{A}$ is $f(v, r)=0$ in Eq. (29). On the apparent horizon, the energy flow across the apparent horizon is $[10,34,37,38]$

$$
\begin{aligned}
\delta Q & =\left.d M_{\mathrm{eff}}\right|_{r_{A}}=\left.A \Psi_{a} d x^{a}\right|_{r=r_{A}} \\
& =A \Psi_{v} d v=-\frac{(n-2) V_{k} r_{A}^{n-3}}{16 \pi G} \dot{f}\left(r_{A}\right) d v .
\end{aligned}
$$

On the other hand, the temperature of generalized dynamical solution is $T=\frac{\kappa}{2 \pi}$, where the surface gravity $\kappa$ defined on the apparent horizon is $\kappa=D_{a} D^{a} r=$ $\frac{1}{2 \sqrt{-h}} \frac{\partial}{\partial x^{\mu}}\left(\sqrt{-h} h^{\mu \nu} \partial_{v} r\right)=f^{\prime}\left(r_{A}\right) / 2$ [10,34-38]. Here, $D_{a}$ is the covariant derivative associated with metric $h_{a b}$. In addition, the entropy of the apparent horizon is $S=\frac{A}{4 G}=$ $\frac{V_{k} r_{A}^{n-2}}{4 G}$ [18]. Therefore,

$$
T d S=\frac{\kappa}{2 \pi} d S=\frac{(n-2) V_{k} r_{A}^{n-3}}{16 \pi G} f^{\prime}\left(r_{A}\right) \dot{r}_{A} d v .
$$

After using the simple relation $f^{\prime}\left(r_{A}\right) \dot{r}_{A}=-\dot{f}\left(r_{A}\right)$ derived from $f\left(r_{A}, v\right)=0$, we can easily obtain that the usual Clausius relation $\delta Q=T d S$ does hold on the apparent horizon of the generalized dynamical solution, which indicates that the dRGT massive gravity is an equilibrium state [44]. Note that this result is consistent with the investigation in Ref. [10] by taking the FRW universe into account. In addition, it should be emphasized that the usual Clausius relation $\delta Q=T d S$ does not always hold on the apparent horizon. For example, the usual Clausius relation does not hold for the $f(R)$ gravity, which can be treated as the effects of the nonequilibrium of the spacetime $[41,43,44]$. Therefore, after taking Eq. (41) and the Clausius relation into account, the unified first law in Eq. (35) on the apparent horizon can be rewritten as

$$
d M_{\mathrm{eff}}=T d S+W d V,
$$

which is just the first law of thermodynamics for the generalized Vaidya-like solution. Note that the work density $W$ in Eq. (42) is nonzero for the generalized Vaidya-like solution, which makes another difference from the generalized Vaidya solution, of which $W=0$.

\section{GENERALIZED MISNER-SHARP MASS FOR THE $N$-DIMENSIONAL MASSIVE GRAVITY}

Note that the Misner-Sharp mass is a quantity depending on not only the symmetry in the solution, i.e., usually just defined in a spacetime with a maximally symmetric subspace, but also the underlying gravity theory. Hence, the existence of the Minser-Sharp mass in a special solution with a maximally symmetric subspace does not always guarantee its existence in the gravity for the general solutions with the same maximally symmetric subspace, for example, the $f(R)$ gravity $[41,42]$. Therefore, we should further investigate the existence of the MisnerSharp mass in a general spacetime with a maximally symmetric subspace. Moreover, in order to investigate the generalized Misner-Sharp mass for the $n$-dimensional dRGT massive gravity, we usually write down the more general metric ansatz in a double-null coordinate as follows:

$$
d s^{2}=-2 e^{-\varphi(u, v)} d u d v+r^{2}(u, v) \gamma_{i j} d x^{i} d x^{j} .
$$

Here, $\gamma_{i j}$ is the metric on the maximally symmetric subspace same as in Eq. (2). In the coordinates (43), the rhs of Eq. (35) reads

$$
A \Psi_{a} d x^{a}+W d V=A(u, v) d u+B(u, v) d v,
$$

where

$$
\begin{aligned}
& A(u, v)=V_{k} r^{n-2} e^{\varphi}\left(r,{ }_{u} T_{u v}-r,{ }_{v} T_{u u}\right), \\
& B(u, v)=V_{k} r^{n-2} e^{\varphi}\left(r,{ }_{v} T_{u v}-r,{ }_{u} T_{v v}\right) .
\end{aligned}
$$

Here, a comma denotes a partial derivative. Substituting Eq. (35) into Eq. (44), we reach

$$
F \equiv d M_{\text {eff }}=A(u, v) d u+B(u, v) d v .
$$

The components of the field equation (5) in the coordinates (43) read 


$$
\begin{aligned}
8 \pi G T_{u u}= & -(n-2) \frac{\varphi,{ }_{u} r,_{u}+r,{ }_{u u}}{r}, \\
8 \pi G T_{v v}= & -(n-2) \frac{\varphi,{ }_{v} r,_{v}+r,{ }_{v v}}{r}, \\
8 \pi G T_{u v}= & \frac{-\Lambda}{e^{\varphi}}+\frac{n-2}{2 e^{\varphi} r}\left(2 r,{ }_{u v} e^{\varphi}+c_{1} c_{0} m^{2}\right)+\frac{(n-2)(n-3)\left(k+2 e^{\varphi} r,_{u} r,_{v}+c_{2} c_{0}^{2} m^{2}\right)}{2 e^{\varphi} r^{2}} \\
& +\frac{(n-2)(n-3)(n-4) c_{3} c_{0}^{3} m^{2}}{2 e^{\varphi} r^{3}}+\frac{(n-2)(n-3)(n-4)(n-5) c_{4} c_{0}^{4} m^{2}}{2 e^{\varphi} r^{4}} .
\end{aligned}
$$

Obviously, a well-defined $M_{\text {eff }}$ in Eq. (47) requires $F$ is a closed form $d F=0$, which means

$$
A_{, v} d v \wedge d u+B_{, u} d u \wedge d v=0 .
$$

Then, we obtain the constraint for a well-defined $M_{\text {eff }}$,

$$
A_{, v}=B_{, u} .
$$

Substituting Eq. (48) into Eqs. (45) and (46), we obtain

$$
\begin{aligned}
& A(u, v)=\frac{V_{k}}{8 \pi G}\left[-\Lambda r_{,} r^{n-2}+(n-2) r^{n-3} e^{\varphi} r,_{u} r,{ }_{v u}+\frac{k}{2}(n-2)(n-3) r^{n-4} r{ }_{u}+(n-2)(n-3) e^{\varphi} r,{ }_{v} r{ }_{, u}^{2} r^{n-4}\right. \\
& +e^{\varphi} r^{n-3}(n-2)\left(r,{ }_{u} r,_{v}+r,{ }_{v} r,_{u v}\right)+\frac{(n-2) r^{n-3} r{ }_{,} c_{1} c_{0} m^{2}}{2}+\frac{(n-2)(n-3) r^{n-4} r_{, u} c_{2} c_{0}^{2} m^{2}}{2} \\
& \left.+\frac{(n-2)(n-3)(n-4) r^{n-5} r_{,} c_{3} c_{0}^{3} m^{2}}{2}+\frac{(n-2)(n-3)(n-4)(n-5) r^{n-6} r_{{ }_{u}} c_{4} c_{0}^{4} m^{2}}{2}\right], \\
& B(u, v)=\frac{V_{k}}{8 \pi G}\left[-\Lambda r,{ }_{v} r^{n-2}+(n-2) r^{n-3} e^{\varphi} r,{ }_{v} r,{ }_{v u}+\frac{k}{2}(n-2)(n-3) r^{n-4} r,{ }_{v}+(n-2)(n-3) e^{\varphi} r,{ }_{u} r_{,}{ }_{v} r^{n-4}\right. \\
& +e^{\varphi} r^{n-3}(n-2)\left(r,{ }_{v} r{ }_{u}+r,{ }_{u} r_{u v}\right)+\frac{(n-2) r^{n-3} r{ }_{v} c_{1} c_{0} m^{2}}{2}+\frac{(n-2)(n-3) r^{n-4} r,_{v} c_{2} c_{0}^{2} m^{2}}{2} \\
& \left.+\frac{(n-2)(n-3)(n-4) r^{n-5} r,{ }_{v} c_{3} c_{0}^{3} m^{2}}{2}+\frac{(n-2)(n-3)(n-4)(n-5) r^{n-6} r,{ }_{v} c_{4} c_{0}^{4} m^{2}}{2}\right] .
\end{aligned}
$$

Using the above explicit forms of $A(u, v)$ and $B(u, v)$, we find that the above constraint is automatically satisfied for the $n$-dimensional dRGT massive gravity, which guarantees that $M_{\text {eff }}$ is well defined. Thus, directly integrating (35) presents the generalized Misner-Sharp mass in the $n$-dimensional dRGT massive gravity,

$$
\begin{aligned}
M_{\mathrm{eff}} & =\int A(u, v) d u+\int\left[B(u, v)-\frac{\partial}{\partial v} \int A(u, v) d u\right] d v \\
& =\frac{V_{k}(n-2)}{16 \pi G} r^{n-3}\left[\frac{r^{2}}{\ell^{2}}+k+2 e^{\varphi} r_{,} r_{,}+\frac{c_{0} c_{1} m^{2}}{n-2} r+c_{0}^{2} c_{2} m^{2}+\frac{(n-3) c_{0}^{3} c_{3} m^{2}}{r}+\frac{(n-3)(n-4) c_{0}^{4} c_{4} m^{2}}{r^{2}}\right] .
\end{aligned}
$$

Note that, here, the second term in the first line of Eq. (52) in fact vanishes, and we have fixed an integration constant so that $M_{\text {eff }}$ reduces to the Misner-Sharp mass in the Einstein gravity when the graviton mass parameter $m$ goes to zero. Furthermore, the above generalized Misner-Sharp mass can be rewritten in a covariant form as

$$
M_{\mathrm{eff}}=\frac{V_{k}(n-2)}{16 \pi G} r^{n-3}\left[\left(k-h^{a b} \partial_{a} r \partial_{b} r\right)+\frac{r^{2}}{\ell^{2}}+\frac{c_{0} c_{1} m^{2}}{n-2} r+c_{0}^{2} c_{2} m^{2}+\frac{(n-3) c_{0}^{3} c_{3} m^{2}}{r}+\frac{(n-3)(n-4) c_{0}^{4} c_{4} m^{2}}{r^{2}}\right] .
$$

For the special case in the above generalized dynamical solution (29), one can check that the result in Eq. (39) is consistent with the generalized Misner-Sharp mass in Eq. (53). And Eq. (53) is the general definition of the generalized Misner-Sharp mass in the $n$-dimensional spacetime with maximally symmetric subspace in the dRGT massive gravity. 


\section{CONCLUSION AND DISCUSSION}

In this paper, through considering the pure radiation and a more general case as the matter fields, we obtain the generalized dynamical solutions in the $n$-dimensional dRGT massive gravity, which naturally includes the generalized Vaidya solution. By using the unified first law and the Misner-Sharp mass, we investigate thermodynamics for these solutions. Besides obtaining the first law of thermodynamics for these generalized dynamical solutions on the apparent horizon, we also check that the generalized Misner-Sharp mass exists for them. Generally, a solution has a much higher symmetry than the theory itself. The existence of the Misner-Sharp mass in a special solution does not imply the existence of it in the general case. For example, the Misner-Sharp mass exists in the FRW solutions and static solutions in $f(R)$ gravity. However, it does not always exist in a general spherically symmetric spacetime in $f(R)$ gravity. In view of this situation, we further investigate the generalized Misner-Sharp by taking the general metric ansatz and matter field into account and find that the generalized Misner-Sharp mass really exists in a covariant form.

Note that in the massive gravity theory a reference metric is required. However, the theory itself does not determine the concrete form of the reference metric. This uncertainty makes the theory become arbitrary in some degree, while it delivers extra conveniences in some cases. For example, there is no Schwarzschild solution in the unitary gauge (Minkowskian reference metric), and thus to match the tests in the Solar System, a chameleon mechanism is necessary. Recently, Li et al. found that the Schwarzschild solution can be obtained if one gives up the unitary gauge [46]. Other solutions have also been found by choosing a different reference metric, for example, the rotating black hole solution in the dRGT massive gravity [47]. Therefore, it is an interesting issue to find other solutions in the dRGT massive gravity by considering different reference metrics.

In addition, it was found recently that the dynamics of black holes and black branes are greatly simplified in the limit of a large number of spacetime dimensions $N$ [48]. Therefore, more properties for the black holes and black branes in the large- $N$ limit will also be an interesting issue to further investigate. Furthermore, according to the AdS/CFT correspondence, the Vaidya dynamical black branes in Eq. (22) can be related to the thermalization processes of the strongly coupled fields [49,50], i.e., thermalization processes of the quark-gluon plasma produced in ultrarelativistic heavy-ion collisions at the Relativistic Heavy Ion Collider and the (LHC). Therefore, the underlying dual physics of our Vaidya-like dynamical black brane in Eq. (29) is also an interesting issue to be explored further.

\section{ACKNOWLEDGMENTS}

This work is supported by the National Natural Science Foundation of China (Grants No. 11575083, No. 11565017, No. 11105004, No. 11075106, and No. 11275128), the Program for Professor of Special Appointment (Eastern Scholar) at Shanghai Institutions of Higher Learning, National Education Foundation of China under Grant No. 200931271104, the Fundamental Research Funds for the Central Universities (Grant No. NS2015073), and the Open Project Program of State Key Laboratory of Theoretical Physics, Institute of Theoretical Physics, Chinese Academy of Sciences, China (Grant No. Y5KF161CJ1). In addition, Y. P. Hu is very thankful for the support from the Sino-Dutch scholarship program under the China Scholarship Council (CSC).
[1] M. Fierz and W. Pauli, Proc. R. Soc. A 173, 211 (1939).

[2] K. Hinterbichler, Rev. Mod. Phys. 84, 671 (2012).

[3] D. G. Boulware and S. Deser, Phys. Rev. D 6, 3368 (1972).

[4] C. de Rham, Living Rev. Relativ. 17, 7 (2014).

[5] C. de Rham and G. Gabadadze, Phys. Rev. D 82, 044020 (2010).

[6] C. de Rham, G. Gabadadze, and A. J. Tolley, Phys. Rev. Lett. 106, 231101 (2011),

[7] S. F. Hassan and R. A. Rosen, Phys. Rev. Lett. 108, 041101 (2012),

[8] S. F. Hassan, R. A. Rosen, and A. Schmidt-May, J. High Energy Phys. 02 (2012) 026.

[9] D. Vegh, arXiv:1301.0537.

[10] Y. P. Hu and H. Zhang, Phys. Rev. D 92, 024006 (2015).

[11] H. Zhang and X. Z. Li, Phys. Rev. D 93, 124039 (2016).
[12] J. M. Maldacena, Adv. Theor. Math. Phys. 2, 231 (1998); Int. J. Theor. Phys. 38, 1113 (1999).

[13] S. S. Gubser, I. R. Klebanov, and A. M. Polyakov, Phys. Lett. B 428, 105 (1998).

[14] E. Witten, Adv. Theor. Math. Phys. 2, 253 (1998).

[15] M. Blake, D. Tong, and D. Vegh, Phys. Rev. Lett. 112, 071602 (2014).

[16] A. Amoretti, A. Braggio, N. Maggiore, N. Magnoli, and D. Musso, J. High Energy Phys. 09 (2014) 160.

[17] Y. P. Hu, H. F. Li, H. B. Zeng, and H. Q. Zhang, Phys. Rev. D 93, 104009 (2016).

[18] R. G. Cai, Y. P. Hu, Q. Y. Pan, and Y. L. Zhang, Phys. Rev. D 91, 024032 (2015).

[19] S. H. Hendi, S. Panahiyan, and B. E. Panah, J. High Energy Phys. 01 (2016) 129; S. H. Hendi, B.E. Panah, and 
S. Panahiyan, J. High Energy Phys. 05 (2016) 029; S. H. Hendi, N. Riazi, and S. Panahiyan, arXiv:1610.01505.

[20] S. H. Hendi, B. E. Panah, and S. Panahiyan, J. High Energy Phys. 11 (2015) 157; Classical Quantum Gravity 33, 235007 (2016).

[21] Y. P. Hu, X. X. Zeng, and H. Q. Zhang, Phys. Lett. B 765, 120 (2017).

[22] M. S. Volkov, Classical Quantum Gravity 30, 184009 (2013).

[23] G. Tasinato, K. Koyama, and G. Niz, Classical Quantum Gravity 30, 184002 (2013).

[24] E. Babichev and R. Brito, Classical Quantum Gravity 32, 154001 (2015).

[25] J. Xu, L. M. Cao, and Y. P. Hu, Phys. Rev. D 91, 124033 (2015).

[26] L. M. Cao and Y. Peng, Phys. Rev. D 92, 124052 (2015); L. M. Cao, Y. Peng, and Y. L. Zhang, Phys. Rev. D 93, 124015 (2016).

[27] R. A. Davison, Phys. Rev. D 88, 086003 (2013).

[28] M. Blake and D. Tong, Phys. Rev. D 88, 106004 (2013).

[29] R. A. Davison, K. Schalm, and J. Zaanen, Phys. Rev. B 89, 245116 (2014).

[30] A. Adams, D. A. Roberts, and O. Saremi, Phys. Rev. D 91, 046003 (2015).

[31] T. Q. Do, Phys. Rev. D 93, 104003 (2016); 94, 044022 (2016).

[32] P. C. Vaidya, Proc. Indian Acad. Sci. (Math. Sci.) 33, 264 (1951); R. W. Lindquist, R. A. Schwartz, and C. W. Misner, Phys. Rev. 137, B1364 (1965).

[33] A. E. Dominguez and E. Gallo, Phys. Rev. D 73, 064018 (2006).

[34] R. G. Cai, L. M. Cao, Y. P. Hu, and S. P. Kim, Phys. Rev. D 78, 124012 (2008).

[35] S. A. Hayward, Phys. Rev. D 49, 6467 (1994); 49, 831 (1994); 53, 1938 (1996); Classical Quantum Gravity 15, 3147 (1998).

[36] H. Maeda and M. Nozawa, Phys. Rev. D 77, 064031 (2008).
[37] R. G. Cai and S. P. Kim, J. High Energy Phys. 02 (2005) 050.

[38] R. G. Cai, L. M. Cao, and Y. P. Hu, J. High Energy Phys. 08 (2008) 090.

[39] H. Zhang, Universe 3, 30 (2015).

[40] H. Zhang, S. Hayward, X. H. Zhai, and X. Z. Li, Phys. Rev. D 89, 064052 (2014); H. Zhang and X. Z. Li, Phys. Lett. B 737, 395 (2014); H. Zhang, D.-J. Liu, and X.-Z. Li, Phys. Rev. D 90, 124051 (2014); D. He and Q.-y. Cai, Sci. China Phys. Mech Astron. 60, 04011 (2017); H. W. Tan, J. B. Yang, T. M. He, and J. Y. Zhang, Commun. Theor. Phys. 67, 41 (2017).

[41] R. G. Cai, L. M. Cao, Y. P. Hu, and N. Ohta, Phys. Rev. D 80, 104016 (2009).

[42] H. Zhang, Y. Hu, and X. Z. Li, Phys. Rev. D 90, 024062 (2014).

[43] M. Akbar and R. G. Cai, Phys. Rev. D 75, 084003 (2007); Phys. Lett. B 635, 7 (2006); 648, 243 (2007).

[44] C. Eling, R. Guedens, and T. Jacobson, Phys. Rev. Lett. 96, 121301 (2006); T. Jacobson, Phys. Rev. Lett. 75, 1260 (1995).

[45] A. Wang and Y. Wu, Gen. Relativ. Gravit. 31, 107 (1999).

[46] P. Li, X. z. Li, and P. Xi, Phys. Rev. D 93, 064040 (2016); Classical Quantum Gravity 33, 115004 (2016).

[47] E. Babichev and A. Fabbri, Phys. Rev. D 90, 084019 (2014).

[48] R. Emparan, R. Suzuki, and K. Tanabe, Phys. Rev. Lett. 115, 091102 (2015); R. Emparan, K. Izumi, R. Luna, R. Suzuki, and K. Tanabe, J. High Energy Phys. 06 (2016) 117.

[49] V. Balasubramanian, A. Bernamonti, J. de Boer, N. Copland, B. Craps, E. Keski-Vakkuri, B. Müller, A. Schäfer, M. Shigemori, and W. Staessens, Phys. Rev. Lett. 106, 191601 (2011).

[50] V. Balasubramanian, A. Bernamonti, J. de Boer, N. Copland, B. Craps, E. Keski-Vakkuri, B. Müller, A. Schäfer, M. Shigemori, and W. Staessens, Phys. Rev. D 84, 026010 (2011); V. Balasubramanian and S. F. Ross, Phys. Rev. D 61, 044007 (2000). 